\title{
Saliva as a Potential Diagnostic Tool for SARS-CoV-2
}

\author{
Aakriti Khemka1 ${ }^{1}$ Manpreet Arora ${ }^{2}$, Aparna Dave ${ }^{3}$, Pulin Saluja ${ }^{4}$, Radhika Rai ${ }^{5}$ \\ 1, 2, 3, 4, 5 Department of Oral Pathology \& Microbiology, SGT Dental College, Gurugram, Haryana, India.
}

\section{ABSTRACT}

\section{BACKGROUND}

COVID 19 has been labelled as a global pandemic by the World Health Organization (WHO). The sudden rise in death toll and devastation associated with it has put the entire infrastructure, economy and health sector to test. The only way for the prevention and control of this infectious disease is rapid and accurate screening of masses. Public Health Authorities mainly use antibody testing in hot spots using a mix of RT-PCR and antibody testing nasopharyngeal and orpharyngeal swabs. Considering the potential risk factors, constraints of time, cost and manpower, mass screening for COVID is not possible through nasopharyngeal and oropharyngeal swabs alone. Hence, to search an alternate method to diagnose for the initial screening of patients is the need of the hour globally. Saliva can also be used as one of the diagnostic modalities for coronavirus, helping in the rapid testing of individuals at home or at hospital. The article intends to explain the evidence regarding the reliability of saliva as a diagnostic specimen in COVID-19 patients and demonstrates the association and potential of detecting novel coronavirus in saliva of patients and how its implication in future can aid in diagnosis as a non - invasive diagnostic modality.

\section{KEY WORDS}

COVID 19, Throat Swab, Nasopharyngeal Swab, Saliva, Diagnostic Fluid
Corresponding Author: Dr. Manpreet Arora, Professor, Department of Oral Pathology \& Microbiology, SGT Dental College, Gurugram, Haryana, India.

E-mail:manpreet.arora@sgtuniversity.org

DOI: $10.14260 / j e m d s / 2021 / 415$

How to Cite This Article:

Khemka A, Arora M, Dave A, et al. Saliva as a potential diagnostic tool for SARS-CoV-2. J Evolution Med Dent Sci 2021;10(27): 2029-2033, DOI:

10.14260/jemds/2021/415

Submission 10-02-2021,

Peer Review 03-05-2021

Acceptance 10-05-2021,

Published 05-07-2021.

Copyright (C) 2021 Aakriti Khemka et al. This is an open access article distributed under Creative Commons Attribution License [Attribution 4.0 International (CC BY 4.0)] 


\section{BACKGROUND}

The Covid 19 pandemic has been considered as the most crucial global health calamity of the century and the greatest challenge that humankind has ever faced since the $2^{\text {nd }}$ World War. It has been labelled as a global pandemic by the World Health Organization and has been declared as a public health emergency of international concern. ${ }^{1}$

Compared with leading causes of death from the same period in 2018, this was the third leading cause of death for children and adults (ranking only behind heart disease and cancer), thus sparing no age group. ${ }^{2}$ Besides the enormous number of deaths and sufferings, the pandemic has posed innumerable economic, environmental and social challenges to the entire human population. In the absence of any definite therapy against COVID-19, the resilience of the health care infrastructure and health professionals has been put to test. ${ }^{3}$

In the last twenty years, several viral epidemics like severe acute respiratory syndrome coronavirus (SARS-CoV), H1N1 influenza, have been reported. In the recent past, the East respiratory syndrome coronavirus (MERS-CoV) was identified and was first reported in Saudi Arabia. ${ }^{4}$ But the novel coronavirus (2019 - nCoV) or the severe acute respiratory syndrome corona virus 2 (SARS-CoV-2) which essentially causes severe acute respiratory syndrome along with many other complications shall always be remembered as a worldwide pandemic.

An epidemic of extreme respiratory deterrence, pneumonia and shortness of breath, the SARS-CoV-2 viral infection began in Wuhan, Hubei Province, China in December 2019, and rapidly spread across China and beyond, with human to human transmission. On February 12, 2020, World Health Organization officially named the new coronavirus disease as coronavirus disease 19 (COVID-19). ${ }^{5}$

The term Corona Virus originates from the Latin word corona, meaning "crown" or "wreath", itself borrowing from

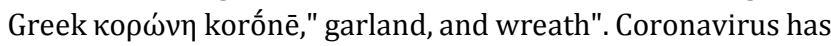
a series of crown - like spikes on its surface. The virus has been named novel as it has not been identified previously. The exact source of the 2019 novel coronavirus has not been identified but it is suggested that the virus likely emerged from an animal source. ${ }^{6}$

Human coronavirus was first observed and studied by June Almeida and David Tyrrell. There are four separate families, the alpha, beta, gamma, and delta coronaviruses. Alpha and beta coronaviruses appear to come from mammals, especially from bats, whereas gamma and delta coronaviruses derive from pigs and birds. The genome length ranges from $26 \mathrm{~kb}$ to $32 \mathrm{~kb}$. Among the seven subtypes of coronavirus that may have the ability to infect humans, beta - coronavirus is considered to be the most dangerous; this is the one that causes significant morbidity and mortality in humans. SARS-CoV-2 virus belongs to the genus beta - coronavirus. Coronaviruses are enveloped positive sense RNA viruses.

The mode of transmission for COVID-19 is primarily through respiratory droplets, by direct human - to human contact and touching the objects that have been contaminated by an infected person. ${ }^{6}$ Once inside the body, SARS-CoV- 2 uses Angiotensin - converting enzyme 2 (ACE2) receptor for entry into the cells.

SARS-CoV-2 uses ACE2 receptor for entry into the cell in our body. All tissues and organs of the body that express ACE2 receptors are prone to face coronavirus invasion and may present with various symptoms. In previous experimental studies, ACE2 receptors were highly expressed in the salivary glands as compared to the lungs. Sites in the oral cavity which have abundant number of ACE2 receptors include tongue and floor of the mouth. ${ }^{7}$ ACE 2 receptor is the primary site of action for coronavirus and salivary glands have an abundant number of these receptors. Moreover, the presence of ACE2 receptors in salivary glands indicate that these glands are a potential target for inflammation by SARS-CoV-2. Thus, it shows a positive correlation between the COVID-19 virus and its presence in saliva. ${ }^{8}$

In past also, saliva has been known to harbor many viruses, presence of which aid in detection of many pathology. Previously, viruses such as Zika virus, Epstein - Barr virus, Herpes Simplex virus, Chikungunya and Ebola viruses have also been inoculated from saliva of the infected patients, indicating interaction of various salivary biochemicals with the virus. ${ }^{8}$

Animals infected with corona virus, have also shown the presence of the virus in their saliva. In Rhesus Macaques, the epithelial cells lining the salivary glands were early targets of SARS-CoV-2. It has also been reported that saliva is a reservoir for coronavirus in asymptomatic patients, who can also transmit the virus to other healthy individuals. This process is facilitated by a serine protease, TMPRSS2 which also causes viral activation by cleavage of $S$ glycoprotein.

ACE2 is abundantly expressed in the epithelial cells of the oral mucosa, with higher expression in the tongue as compared to the buccal and gingival tissues. This receptor plays an important role in allowing SARS-CoV-2 to enter the cells then to eventually gain an entry into the saliva. Another route of entry into saliva is via the liquid droplets which are loaded with SARS-CoV-2 in the lower and upper respiratory tract. When patient coughs the virus reaches the oral cavity along with the droplets and gains entry into saliva. SARS-CoV2 can also enter the oral cavity via gingival crevicular fluid. ${ }^{8}$

Braz - Silva et al, $^{9}$ also proposed similar findings stating that oral epithelial cells of frequently shed saliva can be an alternative medium for detection of viruses.

Mode of spread of this virus is through oral and nasal secretions, cough, sneeze, and droplet inhalation or via contact transmission like ocular contact, saliva, mucous membranes of the nose and eyes. Transmission through salivary bioaerosols poses a significant danger to healthcare workers that operate on the brink of the face and oral cavities, like dental practitioners; oral - maxillofacial surgeons; ear, nose, and throat (ENT; otorhinolaryngology) surgeons; and ophthalmologists. ${ }^{10}$

The incubation time of virus might be generally within 3 to 7 days median 5.1 days and up to 2 weeks. Majority of the patients infected with SARS-CoV-2 developed mild symptoms like dry cough, pharyngitis, and fever. Some cases have developed various fatal complications including organ failure, septic shock, pulmonary oedema, severe pneumonia, and Acute Respiratory Distress Syndrome (ARDS). Sometime patients may present with sputum production, hemoptysis, headache and gastrointestinal symptoms such as diarrhoea, nausea and vomiting. ${ }^{11}$

Virus shows oral symptoms like dry mouth, amblygeustia, dryness, and inflammation of the mouth and submandibular lymph nodes enlargement. Hence oral symptoms and hygiene 
can play an important role in diagnosing and preventing COVID-19 and requires further exploration. ${ }^{12}$

Wang et al. ${ }^{13}$ in his study stated that, before lung lesions are seen, SARS-CoV are often found within the saliva. It is believed that expression ACE receptors in minor salivary glands is higher than lungs. It could be possible that a person does not develop respiratory symptoms but has virus in saliva and these patients could be asymptomatic carriers.

Hence a diagnosis at right time will reduce the distress that the patient has to undergo. Thus for controlling the spread of infection, it is advisable to have testing done on more number of individuals including asymptomatic cases. It is a known fact that asymptomatic cases are larger in number and could be more responsible for the spread of disease. Therefore, the main strategy for controlling the pandemic depends on testing as many individuals as possible to avoid the risk of transmission to other patients and health care professionals, including transmission from asymptomatic people, who account for approximately $79 \%$ of the contagion. ${ }^{14}$

Public Health Authorities mainly use antibody testing in hot spots using a mix of RT - PCR and antibody testing.

Suspected patient's nasopharyngeal and oropharyngeal swabs are collected for detection of coronavirus. The collected specimens are then tested by using real - time RT - PCR. ${ }^{15}$ Nasopharyngeal and oropharyngeal swabs are considered to be sensitive for SARS-CoV-2 detection, but it is important to collect the specimens at the right time. ${ }^{16}$

Therefore, for screening of patients, blood samples and nasophayryngeal and oropharyngeal swab samples are required. But these tests have a lot of limitations and drawbacks, such as the discomfort for the patient and the need for the intervention of a healthcare worker in a disease with a high risk of nosocomial transmission. These collection systems can also induce coughing and sneezing, generating aerosol, which can transmit the virus. In addition, in cases of thrombocytopenia or any other coagulation disorder, this procedure can cause bleeding. These drawbacks can limit the use of swabs, especially in serial monitoring or mass test programs. Sputum has also been proposed as a non - invasive lower respiratory tract specimen, but $72 \%$ of COVID-19 patients were not able to produce it for collection. The difficulty of obtaining sputum also has been described in SARSCoV, a virus with many similarities with the Covid - 2019, especially at early stages of infection, when no cough or only dry cough is present. ${ }^{17}$

Hence, considering the potential risk factors and constraints of time, cost and manpower, mass screening for COVID is not possible through nasopharyngeal and oropharyngeal swabs alone. As a result, only a small proportion of the part of the population has access to rapid testing, while COVID-19 testing should be widely available. Therefore, search for an alternate method to diagnose for the initial screening of patients is the need of the hour globally.

As already discussed, the presence of the virus has been confirmed in saliva even in asymptomatic carriers owing to its attachment with the ACE 2 receptors. Since time immemorial, saliva has played a key role in the diagnosis of various systemic ailments including various viral infections for use in laboratory and at home. Saliva can also be used as one of the diagnostic modalities for coronavirus and can thus help in the rapid testing of individuals at home or at hospital. ${ }^{8}$
Chen, Yip, et al. 2020 showed no significant difference in the detection rate between NPS and saliva, and the results were in concordance with RT - PCR. ${ }^{15}$ Kim et al. ${ }^{9}$ also postulated that there was no significant difference between NPS and saliva, and the results were in concordance with RT PCR. Study conducted by Jeongs et al. ${ }^{8}$ reported more optimal detection of respiratory viruses in sputum as compared with a nasopharyngeal swab.

Robinson et $\mathrm{al}^{8}$ in his study stated that, saliva is more sensitive for detection of respiratory viruses while Becker et al. in his study noted that nasopharyngeal swab is more sensitive in detecting coronavirus (COVID-19) as compared to saliva specimen. Hence, it can be said that both saliva and nasopharyngeal swab have their own importance as diagnostic modalities.

In some literature, researchers have detected coronavirus just in saliva specimen rather than nasopharyngeal aspirate. Due to this, high validity of diagnosing tests based on saliva specimens for SARS-CoV infections is documented. ${ }^{18}$

Researchers from RUCDR Infinite Biologics at Rutgers University ${ }^{7}$ have successfully validated saliva. Saliva can be utilized for Covid 19 detection, as it is a good source for viral RNA and results obtained are at par that can be seen with swab base collection method.

Saliva can be collected by the patient, even at home, minimizing the exposure of health care workers to nosocomial infections. This also reduces the need for health care personnel and waiting times for sample collection, resulting in easier crowd control regulations in clinical settings and thus avoiding further virus transmission. It is easily accepted by the patients since it is non - painful and non - stressful. Therefore, it can be used for serial samplings and in large scale or epidemiological studies, being especially advantageous in certain populations, such as children. It is easy, fast, and cheap to collect, allowing widespread testing. Moreover, since the method is non invasive, healthcare workers are safe from cross - infection when collecting saliva for diagnosis. Storage and shipping can also be carried out more easily. Furthermore, no additional trained medical staff is required to perform the collection process. Saliva does not clot like blood, so it can be manipulated easily and disposed of as well. ${ }^{17}$

There are several ways by which saliva can be collected such as spitting out, collection with the help of sponge - like device and directly from the salivary gland duct. The cheapest one is spitting out technique, and the saliva sample thus collected also includes nasopharyngeal / oropharyngeal / airway secretions. Sponge - like devices provide relatively more pure saliva, but this technique requires special equipment which is not always widely available. ${ }^{19}$

Early morning saliva has been preferred as a test sample ie before tooth brushing and breakfast, as at night in the supine position, the nasopharyngeal and Broncho pulmonary secretions get collected in the posterior oropharyngeal area. The secretions can be collected by deep cough, spitting or gargling saline.

A Study was conducted by Tajima et al. in which he compared early morning saliva samples with daytime saliva samples and he found that $66.7 \%$ sensitivity in early morning saliva samples when compared with $25.0 \%$ in daytime saliva samples. He also stated that both early morning saliva samples and daytime saliva samples had $100 \%$ specificity. ${ }^{12}$ 
Omid Fakheran et al. ${ }^{18}$ in his review suggested that the use of self - collected saliva as a non - invasive specimen has proper accuracy and reliability regarding detection of SARS-CoV-2 based on RT - PCR technique. This gives qualitative detection of nucleic acid from SARS-CoV-2, within a short run time and with targeting the viral E and N2 gene regions. Detection of both targets alone defines viral presence, while detection of $\mathrm{E}$ alone is considered a presumptive positive result.

However, the use of a standardised method of saliva collection must be in place. As discussed, different methods can be used for saliva collection, such as passive drool, different absorbent materials, or stimulation with citrate. These methods may have, in some cases, interference with selected analytes. In the particular case of the COVID-19, two aspects should be addressed regarding the method of collection. The possible differences between saliva obtained by passive drool or absorbent materials and by clearing the throat should be evaluated. In addition, some patients might not be able to clear the throat effectively to cough out saliva from deep in the throat and, therefore, this could decrease test sensitivity with this collection method, which should be explored.

Instead of direct spitting, the use of a straw or any device that avoids the creation and expansion of drops should be encouraged. The saliva of infected patients can contain viruses that may allow airborne transmission and also by oral droplets and, therefore, should be handled with care. In SARS-CoV, it has been hypothesized that very small particles of the virus can be spread in the air. Therefore, special care should be taken during all the processes of sample collection, management, and analysis to eliminate this potential risk of transmission via contact with saliva droplets or aerosol.

In addition, ideally, the salivary flow should be controlled by establishing a fixed amount of time during which saliva is collected (e.g., one minute). It is worthwhile to indicate that the report of results should be standardised, since in some cases, such as for selected interleukins or alpha - amylase, the way of expressing the values can influence their interpretation. Furthermore, the need for saliva sample centrifugation and the effect of this centrifugation on the analyte of interest should be assessed. Avoiding centrifugation can speed the sample processing, allowing the use of rapid point of care tests; however, centrifugation helps to eliminate components that can interfere with subsequent assays.

Appropriate conditions for sample preservation must also be considered. It is known that the degradation of saliva components can occur under certain storage conditions. As such, it is important to know the best storage conditions for samples that allow the maximum preservation of the analytes to be analysed. Nevertheless, while such data are lacking, it is recommended to keep samples refrigerated until arrival at the lab to be analysed and use $-80{ }^{\circ} \mathrm{C}$ temperatures if the samples are stored.

Another field where saliva may have a major potential for COVID-19 is in screening for immunity. There is scientific evidence that specific antibodies against infectious diseases can be detected in saliva. Salivary IgG and IgM concentrations are much lower than in serum. The presence of IgM and IgG in serum against components of the virus, such as 2019 - $n$ Covid nucleoprotein (NP) and spike protein receptor binding domain (RBD), has been described in COVID-19 patients 10 days or later after the symptom onset. It could be expected that saliva will have a major role to play in COVID-19 diagnosis and monitoring for two main reasons: as long as there is no effective treatment or vaccine and person - to - person transmission has to be minimized, the possibility of identifying immune individuals will be invaluable in defining who and how particular individuals can be relieved from confinement and limited social contact and there is a lot to establish and understand about immunity dynamics during the disease, and how long it remains after the individual has recovered.17

\section{CONCLUSIONS}

In general saliva has been recognized as a promising biological specimen for early detection of diseases. Saliva contains biomolecules such as DNA, RNA, microRNA, protein, and metabolites compared to serum and other body fluids. Saliva sampling has generated great interest in the field of public health. With improvement in recent technology, multiple saliva - based biomarkers have been revealed to correlate with various diseases. Salivary diagnostic tests are economical, noninvasive, easy to use than serum sampling with minimum possibility of cross - infection. Samples are easy to ship and store as compared to serum samples. Saliva doesn't clot and may be handled more efficiently than blood. For strong positive rate of virus identification, saliva from deep throat provides strong positive rate, which could help in early diagnosis of COVID-19. Saliva is often collected by the patients themselves and thereby reduces the danger of infection to the healthcare workers. Salivary diagnostic testing thus offers a convenient and cost - effective mechanism for early - diagnosis of COVID-19. Therefore saliva is a very good alternative sample in the diagnosis of respiratory virus infections particularly for large population - level screenings.

To analyse the potential of detecting coronavirus in salivary samples, more research is required to develop rapid testing methods which can be beneficial for the healthcare professionals, scientists and virologists for early and easy detection. Hence, this area is open for research and more studies should be done to standardise the use of saliva in terms of sample collection and diagnostic tests applied for diagnosis of COVID 19 as well as other viral infections.

Financial or other competing interests: None.

Disclosure forms provided by the authors are available with the full text of this article at jemds.com.

\section{REFERENCES}

[1] Chakraborty I, Maity P. COVID-19 outbreak: migration, effects on society, global environment and prevention. Sci Total Environ 2020;728:138882.

[2] Pal R, Yadav U. COVID-19 pandemic in India: present scenario and a steep climb ahead. J Prim Care Community Health 2020;11:2150132720939402.

[3] Rajgopal T. Antibody testing in the context of COVID-19 and return to work. Indian J Occup Environ Med 2020;24(2):51-4. 
[4] Cascella M, Rajnik M, Cuomo A, et al. Features, evaluation and treatment coronavirus (COVID-19). In: StatPearls. Treasure Island (FL): StatPearls Publishing 2021.

[5] Jan H, Faisal S, Khan A, et al. COVID-19: review of epidemiology and potential treatments against 2019 novel coronavirus. Discoveries (Craivoa) 2020;8(2):e108.

[6] Tyrrell DA, Fielder M. Cold wars: the fight against the common cold. USA: Oxford University Press 2002: p. 96.

[7] Sri Santosh T, Parmar R, Anand H, et al. A review of salivary diagnostics and its potential implication in detection of COVID-19. Cureus 2020;12(4):e7708.

[8] Lal A, Saleem MKM, Ahmed N. Saliva as a diagnostic tool for detection of coronavirus-a review. Journal of Islamabad Medical \& Dental College 2020;9(3):225-32.

[9] Fini MB. Oral saliva and COVID-19. Oral Oncol 2020;108:104821.

[10] Vinayachandran D, Balasubramanian S. Salivary diagnostics in COVID-19: future research implications. J Dent Sci 2020;15(3):364-6.

[11] Martelli-Júnior H, Machado RA, Martelli DRB, et al. Dental journals and coronavirus disease (COVID-19): a current review. Oral Oncol 2020;106:104664.

[12] Kapoor P, Chowdhry A, Kharbanda OP, et al. Exploring salivary diagnostics in COVID-19: a scoping review and research suggestions. BDJ Open 2021;7(1):8.
[13] Xu J, Li Y, Gan F, et al. Salivary glands: potential reservoirs for COVID-19 asymptomatic infection. J Dent Res 2020;99(8):989.

[14] Fernandes LL, Pacheco VB, Borges L, et al. Saliva in the diagnosis of COVID-19: a review and new research directions. J Dent Res 2020;99(13):1435-43.

[15] Corman VM, Landt O, Kaiser M, et al. Detection of 2019 novel coronavirus (2019 - nCoV) by real-time RT-PCR. Euro Surveill 2020;25(3):2000045.

[16] Loeffelholz MJ, Tang YW. Laboratory diagnosis of emerging human coronavirus infections-the state of the art. Emerg Microbes Infect 2020;9(1):747-56.

[17] Ceron JJ, Lamy E, Martinez-Subiela S, et al. Use of saliva for diagnosis and monitoring the SARS-CoV-2: a general perspective. J Clin Med 2020;9(5):1491.

[18] Fakheran 0, Dehghannejad M, Khademi A. Saliva as a diagnostic specimen for detection of SARS-CoV-2 in suspected patients: a scoping review. Infect Dis Poverty 2020;9(1):100.

[19] Sapkota D, Søland TM, Galtung HK, et al. COVID-19 salivary signature: diagnostic and research opportunities. J Clin Pathol 2020;jclinpath-2020-206834. 